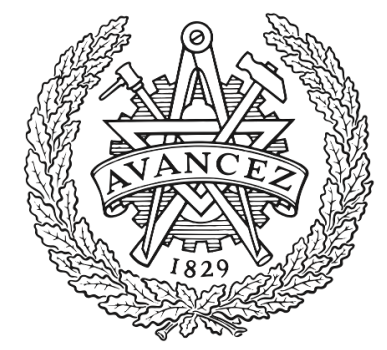

CHALMERS

UNIVERSITY OF TECHNOLOGY

\title{
Quasi-Optical Beamforming Network for Millimeter-Wave Electronically Scanned Array Antennas with 1-Bit Phase Resolution
}

Downloaded from: https://research.chalmers.se, 2023-04-26 12:08 UTC

Citation for the original published paper (version of record):

Vilenskiy, A., Galesloot, E., Zhang, Y. et al (2021). Quasi-Optical Beamforming Network for Millimeter-Wave Electronically Scanned Array Antennas

with 1-Bit Phase Resolution. 15th European Conference on Antennas and Propagation, EuCAP 2021: 1-5. http://dx.doi.org/10.23919/EuCAP51087.2021.9410922

N.B. When citing this work, cite the original published paper. 


\title{
Quasi-Optical Beamforming Network for Millimeter-Wave Electronically Scanned Array Antennas with 1-Bit Phase Resolution
}

\author{
Artem R. Vilenskiy*, Esmé Galesloot ${ }^{\dagger}$, Yingqi Zhang*, Adrianus Bart Smolders ${ }^{\dagger}$, Marianna V. Ivashina* \\ *Electrical Engineering Department, Chalmers University of Technology, 41296 Gothenburg, Sweden \\ artem.vilenskiy@chalmers.se, yingqi@chalmers.se, marianna.ivashina@chalmers.se \\ ${ }^{\dagger}$ Electromagnetics Group, Eindhoven University of Technology, 5612 Eindhoven, The Netherlands \\ e.galesloot@student.tue.nl, A.B.Smolders@tue.nl
}

\begin{abstract}
State-of-the-art design solutions for electronically scanned array antennas are mostly limited to microwave to low mm-wave frequency bands, while the demand for new designs at higher frequencies (i.e. frequencies beyond $100 \mathrm{GHz}$ ) is rapidly growing. We attempt to fill in this knowledge gap by presenting a new linear array antenna architecture as a building block of $2 \mathrm{D}$ arrays that can enable efficient beam steering and a simplified array design. This concept is based on the combination of a low-loss quasi-optical (QO) feed, providing predefined antenna port excitation, with 1-bit phase shifters which are co-integrated with the array antenna elements. In this study, we formulate the array design problem as minimization of the sidelobe level (SLL) through an optimum quasi-randomization of phase errors. An analytical expression for the optimum focal ratio of the QO feed has been derived to establish the relationships between the key design parameters. These results are validated through numerical simulations revealing that the optimum focal ratio leads to the minimum SLL.
\end{abstract}

Index Terms-phased array antenna, quasi-optical beamforming network, 1-bit phase quantization, phase errors.

\section{INTRODUCTION}

An increasing demand in millimeter-wave (mm-wave) radio systems for wireless communication and sensing drives the development of high-performance mm-wave phased array antennas (PAAs) with beam-steering capabilities. In particular, E-, W-, and D-bands have already been widely allocated for communication and radar applications and are being considered for future (e.g. beyond 5G) networks deployment [1]. However, high dissipation losses, components cost, and tight manufacturing tolerances at these frequencies severely restrict suitability of the existing design solutions and manufacturing technologies. These complexities steer the research towards non-conventional PAA architectures, where specific design trade-offs can provide relatively high electrical performance with a simple PAA structure.

Recently, the concept of low-order (1- and 2-bit) phase control was applied to beam-steerable PAAs to realize compact and energy-efficient designs at $<30 \mathrm{GHz}$ bands [2]-[5]. This approach is especially promising for higher frequencies. In fact, at high mm-wave frequencies, the state-of-the-art monolithic digital phase shifters (PSs) typically provide 2.5$3 \mathrm{~dB}$ insertion loss per bit [6]. At the same, the PAA gain loss due to 1-bit phase quantization errors is around $3.8 \mathrm{~dB}$ and almost $1 \mathrm{~dB}$ for 2-bit quantization [7]. Thus, at W-band, 1bit and 2-bit based architectures demonstrate similar antenna gain and efficiency, while the 1-bit PAAs have an advantage of a simpler and more compact design. The key challenge of such designs is phase quantization sidelobes appearing due to periodic PAA aperture phase errors [8], which are especially severe for the 1-bit PAAs. As studied in [7], parasitic mirror lobes occur during beam-steering when a 1-bit PAA is exited with a linear phase and a simple rounding-off method is used to set the PS phase. This effect can be reduced via phase quantization error randomization, as demonstrated in [9].

In this work, we investigate the 1-bit PAA concept for the applications at $\sim 100 \mathrm{GHz}$ bands. A new linear array antenna architecture is proposed as a building block of 2D arrays which can enable efficient beam steering, while overcoming the major physical constraints and power loss at these frequencies.

\section{Proposed 1-Bit PAA ARChitecture}

The proposed linear PAA architecture is illustrated in Fig.1. It includes an array of $N_{x}$ radiating antenna elements with inter-element spacing $d_{x}$. The elements are excited through a planar quasi-optical (QO) beamforming network. This network comprises a QO feed - representing a low-loss alternative to conventional corporate feed networks for mm-wave largescale arrays $\left(N_{x}>10\right)-$ and $N_{x}$ 1-bit PSs which can be co-integrated with the individual array elements. The PS's states differ from element to element between 0 and $180^{\circ}$ values depending on a beam-steering direction. The QO feed is a crucial component for efficient feeding that also realizes a beam-steering functionality in conjunction with the 1-bit PSs. The design principle of such a hybrid (QO feed + PSs) beamforming network can be described in the following way. Due to the 1-bit phase quantization, it is necessary to have a certain nonlinear initial phase distribution at the array ports (see the reference plane at the input ports of the PSs in Fig. 1). This nonlinear distribution is essential to eliminate 


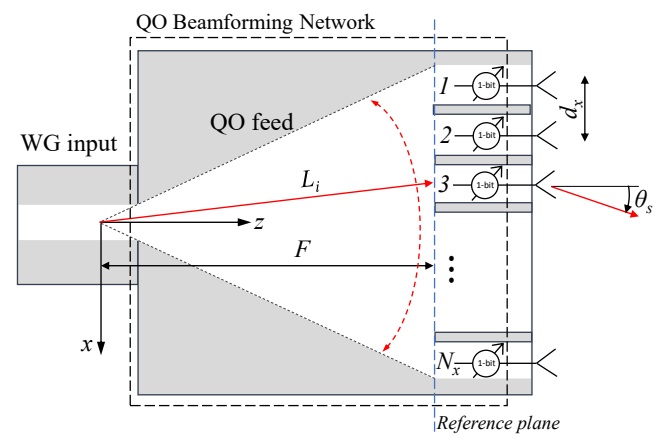

Fig. 1. A 1D sub-array building block of the proposed 1-bit beam-steering phased array architecture at W-band.

the 1-bit parasitic mirror lobes [4], [7]. It can be considered as a phase error quasi-randomization method realizing the performance close to the phase-added method [9]. In hardware, this initial excitation can be implemented by feeding the subarray through a tapered waveguide section with the focal ratio $F /\left(N_{x} d_{x}\right)$ (cf. [10]), where $F$ is the focal length (see Fig. 1).

The main advantage of this architecture is its highlyintegrated and simple design, which can be manufactured in a standard waveguide technology and, as opposed to corporate feed PAAs, conveniently realizes the nonlinear initial phase distribution for high $N_{x}$. In fact, using the space (optical) PAA feeding to deliberately destroy the periodicity of phase quantization errors was proposed a long time ago in [11] and is used in 1- and 2-bit transmitarrays with a focal source [3], [5]. Nevertheless, there are still open questions that we address in this study: (i) What is the optimum initial phase distribution in terms of the lowest 1-bit PAA sidelobe level (SLL)? (ii) What is the optimum $Q O$ feed design realizing the latter?

In [7], these questions were addressed for 1-bit PAAs with a uniform amplitude distribution. In this paper, we extend this approach to non-uniform amplitude distributions and provide an in-depth analysis of the SLL performance showing that the maximum SLL can be effectively reduced by using the optimum QO feed focal ratio.

\section{1-BIT ARRAY FAR FIELD}

\section{A. Radiation Model}

We consider a linear array of $N_{x}$ elements (Fig. 1). Neglecting the edge effects, the array far field can be represented as

$$
\mathbf{E}_{F F}(\theta)=\mathbf{F}_{e}(\theta) \sum_{i=1}^{N_{x}} A_{i} \exp \left(j\left[\Phi_{i}+\varphi_{i}^{\Sigma}\right]\right),
$$

where $\mathbf{F}_{e}$ is the embedded element pattern (azimuthal dependence is omitted); $\Phi_{i}=k_{0} x_{i} \sin (\theta), x_{i}=\left[i-\left(N_{x}+1\right) / 2\right] d_{x}$, $k_{0}=2 \pi / \lambda_{0}$ is the wavenumber, $\lambda_{0}$ is the free-space wavelength; $A_{i}$ and $\varphi_{i}^{\Sigma}$ are the $i$-th element excitation amplitude and phase. The latter can be expressed through the output phase $\varphi_{i}^{Q O}$ at the reference plane and the 1-bit PS phase $\varphi_{i}^{P S}$ :

$$
\varphi_{i}^{\Sigma}=\varphi_{i}^{Q O}+\varphi_{i}^{P S}
$$

On the other hand, $\varphi_{i}^{\Sigma}=\Phi_{i}^{0}+\delta \varphi_{i}$, where $\Phi_{i}^{0}=$ $-k_{0} x_{i} \sin \left(\theta_{s}\right)$ is an ideal element phase, $\theta_{s}$ is a beam-steering angle, $\delta \varphi_{i}$ is a phase quantization error. The excitation through the QO feed is approximated by a cylindrical phase front emanating from the focal center at the distance $F$ from the reference plane (Fig. 1):

$$
\varphi_{i}^{Q O}=-k_{0}\left(\sqrt{x_{i}^{2}+F^{2}}-F\right) .
$$

In (3), we assume the QO feed propagation constant equals $k_{0}$. At the same time, the amplitude distribution is modeled as the cosine-on-a-pedestal function [8] (with the taper parameter $C)$, which was found to be a reasonable approximation for the QO feed with $y$-oriented $E$-field (Fig. 1):

$$
A_{i}=C+(1-C) \cos \left(\pi x_{i} /\left(N_{x} d_{x}\right)\right) .
$$

Since we are dealing with the 1-bit phase quantization, $\varphi_{i}^{P S}$ switches between 0 and $180^{\circ}$ values during beam steering [7], and thus we need to find an optimum value of $F$ that will provide "the best" $\delta \varphi_{i}$ randomization over the PAA aperture.

\section{B. Optimum Quasi-Optical Feed Design}

Let us consider the AF, which is given by the summation factor in (1). Following the procedure used in [7], [12], we can represent the PAA AF in the spectral domain as a superposition of radiation from the continuous apertures with phase distributions $\Phi_{m h}(x)$. Indices $m$ and $h$ denote the grating lobe and phase quantization orders, respectively. Thus, each spectral term $F_{m h}, h \neq 0$, gives the contribution to the AF due to the phase quantization errors. We will herein refer to these terms as phase quantization lobes (PQLs). For electrically large PAAs with $N_{x} d_{x}>>\lambda_{0}$, the stationary phase method can be used to estimate PQL values [7]:

$$
\begin{aligned}
& F_{m h}(\theta)=\frac{(-1)^{m\left(N_{x}-1\right)} \sqrt{2 \pi}}{\sqrt{-j d^{2} \Phi_{m h}\left(x_{0}\right) / d x^{2}}} \frac{A\left(x_{0}\right)}{1+M h} \mathrm{e}^{j \Phi_{m h}\left(x_{0}\right)}, \\
& \Phi_{m h}(x)=\Phi(x)+\Phi^{0}(x)+ \\
& M h\left(\Phi^{0}(x)-\varphi^{Q O}(x)\right)-\frac{2 \pi m x}{d_{x}}, \\
& M h \frac{d \varphi^{Q O}\left(x_{0}\right)}{d x}=k_{0} \sin (\theta)-k_{0} \sin \left(\theta_{s}\right)(1+M h)-\frac{2 \pi m}{d_{x}},
\end{aligned}
$$

where $M=2^{p}$, and $p=1$ is the number of PS bits. In (5)-(7), all continuous functions $(A(x), \varphi(x))$ are equal to the discrete equivalents at array grid points $x_{i}$. We can now require PQL power values to remain constant and equal to $K_{h}^{2}$ for any $m$. This leads to the differential equation

$$
\frac{d^{2} \varphi^{Q O}(x)}{d x^{2}}=-\frac{2 \pi A^{2}(x)}{K_{h}^{2} M|h|(1+M h)^{2}} .
$$

It is now up to us to choose any value of $K_{h}^{2}$. However, from (7) and (8) we can find that decreasing $K_{h}^{2}$ leads to the $F_{m h}$ PQL widening. In [12], it was suggested that the 
PAA SLL can be minimized if PQLs do not overlap. We will employ the same approach here. From (5), we can see that the PQL width is determined by $A\left(x_{0}\right)$, which is non-zero only for $x_{0} \in\left[-N_{x} d_{x} / 2 ; N_{x} d_{x} / 2\right]$. According to (7), PQLs are equidistantly distributed in the $u$-space, where $u=k_{0} \sin (\theta)-$ $k_{0} \sin \left(\theta_{s}\right)(1+M h)$, with the period $2 \pi / d_{x}$. Thus, requiring that the PQL width be equal $2 \pi / d_{x}$ we arrive at

$$
M|h|\left(\frac{d \varphi^{Q O}\left(N_{x} d_{x} / 2\right)}{d x}-\frac{d \varphi^{Q O}\left(-N_{x} d_{x} / 2\right)}{d x}\right)=\frac{2 \pi}{d_{x}} .
$$

Integrating (8) one time and employing (9), we find the optimum $K_{h o p t}^{2}$ :

$$
K_{h o p t}^{2}=\frac{N_{x} d_{x}^{2}}{(1+M h)^{2}}\left[C^{2}+\frac{4 C(1-C)}{\pi}+\frac{(1-C)^{2}}{2}\right] \text {. }
$$

Next, integrating twice both sides of (8) and setting integration constants to 0 we can find the sought-for optimum initial phase distribution $\varphi_{o p t}^{Q O}$ :

$$
\begin{aligned}
& \varphi_{o p t}^{Q O}(x)=-\frac{2 \pi}{K_{h o p t}^{2} M|h|(1+M h)^{2}} \times \\
& {\left[\left(C^{2}+\frac{(1-C)^{2}}{2}\right) \frac{x^{2}}{2}-\frac{2 C(1-C) N_{x}^{2} d_{x}^{2}}{\pi^{2}} \cos \left(\frac{\pi x}{N_{x} d_{x}}\right)\right.} \\
& \left.\quad-\frac{(1-C)^{2} N_{x}^{2} d_{x}^{2}}{8 \pi^{2}} \cos \left(\frac{2 \pi x}{N_{x} d_{x}}\right)\right] .
\end{aligned}
$$

The PQLs with lower $|h|$ have the highest peak values and, thus, affect the SLL to a greater extent. Therefore we consider $|h|=1$. Since the QO feed can physically realise $\varphi^{Q O}$ as described by (3), we need to relate it to (11). To do this, we first represent (3) as $\varphi^{Q O}(x) \approx-k_{0} x^{2} /(2 F), F /\left(N_{x} d_{x}\right)>$ 0.5 , and use the cosine function approximation $\cos (\Theta) \approx 1-$ $\Theta^{2} / 2, \Theta<1$. Then, from (11), we can find the approximate value of the optimum focal distance $F_{o p t}$ :

$$
F_{\text {opt }} \approx \frac{M N_{x} d_{x}^{2}}{\lambda_{0}}\left[C^{2}+\frac{4 C(1-C)}{\pi}+\frac{(1-C)^{2}}{2}\right] .
$$

It should be mentioned that the employed two-term cosine expansion gives a high error for $\Theta>1$. Therefore, (12) tends to underestimate $F_{\text {opt }}$ for small $C$. Finally, we can find the normalized PQL power as $Q_{h}^{2}=K_{h o p t}^{2} / F_{00}^{2}$, where $F_{00}$ is the main lobe field intensity (see (13) in [7]) $F_{00}=\int_{-N_{x} d_{x} / 2}^{N_{x} d_{x} / 2} A(x) d x=N_{x} d_{x}(C+2(1-C) / \pi)$.

$$
Q_{h}^{2}=\frac{1}{N_{x}(1+M h)^{2}} \frac{\left[C^{2}+\frac{4 C(1-C)}{\pi}+\frac{(1-C)^{2}}{2}\right]}{(C+2(1-C) / \pi)^{2}} .
$$

The highest PQL power is observed for $h=-1$. It determines the expected lower bound of the average SLL, which was found to be similar to the average phase error SLL of a PAA with phase errors uniformly distributed between $-\pi / M$ and $+\pi / M$ [9]. Let us consider two special cases.

- Uniform amplitude distribution $(C=1)$ :

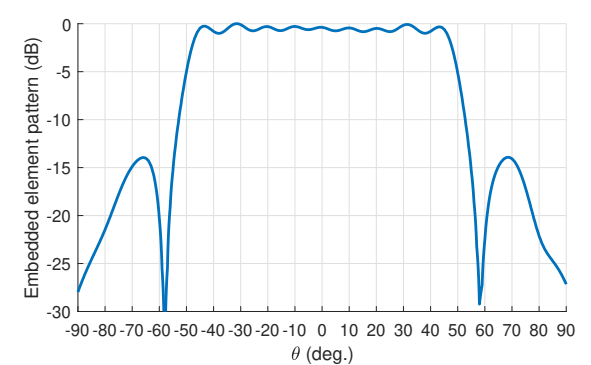

Fig. 2. Normalized embedded element radiation pattern.

$$
F_{\text {opt }} \approx M N_{x} d_{x}^{2} / \lambda_{0}, \quad Q_{-1}^{2}=1 / N_{x}
$$

- Cosine amplitude distribution $(C=0)$ :

$$
F_{\text {opt }} \approx M N_{x} d_{x}^{2} /\left(2 \lambda_{0}\right), \quad Q_{-1}^{2}=\pi^{2} /\left(8 N_{x}\right) .
$$

The last result evidences that the average SLL for the tapered distribution is almost the same as for the uniform one. This is due to the quasi-random nature of PQLs.

\section{NumericAl Simulations}

In this section, we provide the far-field simulated results for linear PAAs, as computed from (1). For all the considered cases of the PAAs, the inter-element spacing is chosen $d_{x}=0.6 \lambda_{0}$ and the embedded element pattern has the shape as shown in Fig. 2. The linear array radiating element has been designed to enable grating lobe-free and impedance-matched beam steering up to $\left|\theta_{s}\right|=45^{\circ}$. The amplitude taper has been computed as $-20 \log \left(A\left(x_{1}\right)\right)$.

The simulated SLL performance for different array parameters is given in Fig. 3 versus the QO feed focal ratio $F /\left(N_{x} d_{x}\right)$. Here, we consider: maximum SLL, first SLL, and mean SLL. Each metric is computed first for each $\theta_{s}$ and then averaged over the $-40 \ldots 40^{\circ}$ beam-steering range. The mean SLLs are relatively invariant with focal ratio variations, and generally depend only on $N_{x}$, as predicted by (13). The computed values, however, are slightly (1-2 dB) higher, which is believed to be due to higher-order PQLs. For electrically large array apertures $\left(N_{x}>10\right)$, the increase in the amplitude taper from 0 to $10 \mathrm{~dB}$ effectively reduces the first SLL. Nevertheless, the two above-mentioned trends are no longer valid for small $N_{x}$ with high taper values, when the PAA aperture is electrically small and PQLs cannot be effectively reduced. On the other hand, such configurations are rarely used for 1-bit PAAs. An important observation in Fig. 3 is that the maximum SLL curves demonstrate well-observable local minima. Positions of these minima are quite close to the expected values of optimum focal ratios (vertical dashed lines) as obtained from (12). The computed optimum value is underestimated for the tapered distributions, as we have discussed in Section III-B. However, the demonstrated results clearly indicate the existence of the optimum focal ratio in terms of maximum SLL minimization. It is also important 

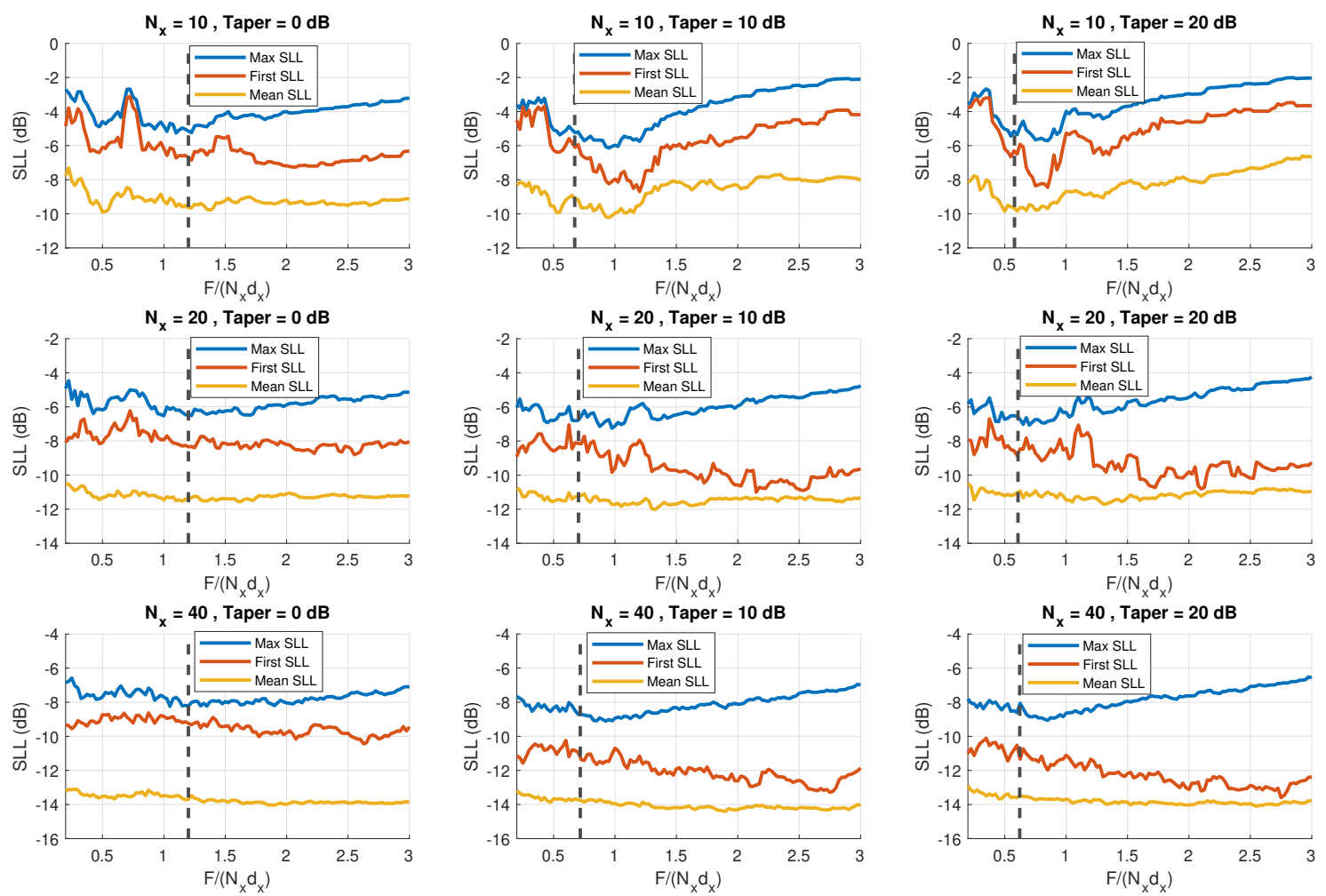

Fig. 3. Averaged (over $\left|\theta_{s}\right| \leq 40^{\circ}$ beam-steering range) SLL performance computed for different configurations of the 1-bit linear phased array antenna versus the focal ratio $F /\left(N_{x} \bar{d}_{x}\right)$ of the quasi-optical feed. Vertical dashed lines show the expected optimum focal ratio values as approximated by (12).

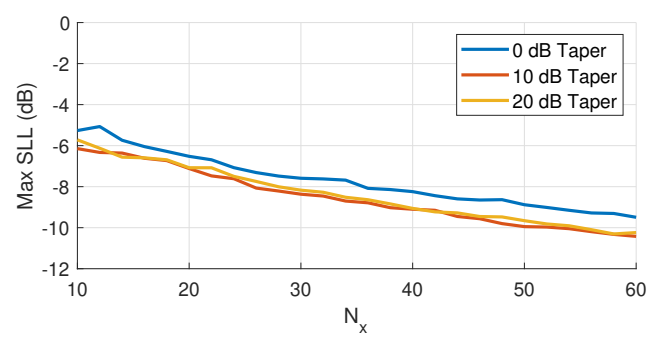

Fig. 4. Averaged (over $\left|\theta_{s}\right| \leq 40^{\circ}$ beam-steering range) maximum SLL versus the number of array elements for different tapers at the optimum (numerically defined) focal ratio $F /\left(N_{x} d_{x}\right)$ of the quasi-optical feed.

that the SLL improvement is more significant for tapered distributions, which is usually the case for the QO feeds. Fig. 4 shows achievable minimized maximum SLL as was found from numerical results analysis for different $N_{x}$ and tapers.

The array gain degradation due to the phase quantization errors was estimated as $\left|\sum_{i=1}^{N_{x}} A_{i}\right|^{2} / \max \left(|A F(\theta)|^{2}\right)$. The averaged results are shown in Fig. 5. With increasing of $N_{x}$, we can observe that the degradation reaches its asymptotic value $\operatorname{sinc}(\pi / 2)$ (see (13) in [7]).

To illustrate the effect of the focal ratio on the far-field performance, the computed PAA radiation patterns are given in Fig. 6 for three configurations: $N_{x}=10,20,40$ with Taper $=$ $0,10,20 \mathrm{~dB}$, respectively. Each configuration is considered

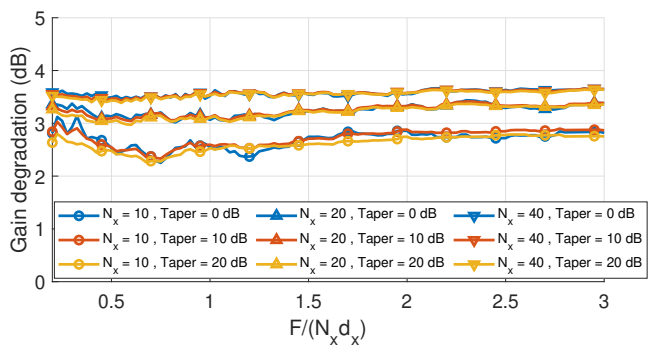

Fig. 5. Averaged (over $\left|\theta_{s}\right| \leq 40^{\circ}$ beam-steering range) gain degradation due to phase quantization errors for different array configurations versus the focal ratio $F /\left(N_{x} d_{x}\right)$ of the quasi-optical feed.

for the optimum (left column, as found from Fig. 3), lower, and higher $F /\left(N_{x} d_{x}\right)$. It can be observed that while the mean SLL remains almost identical, the optimum focal ratios provide balanced SLL distributions over the entire visible angular space, and thus the maximum SLL is effectively reduced for the optimum cases. We, therefore, can summarise that the QO feed, providing the excitation phase distribution (3), indeed has the optimum focal ratio that depends on the distribution taper and realizes the lowest maximum SLL performance.

\section{CONCLUSION}

We have considered the optimum QO feed design for the linear PAA with the 1-bit phase resolution. The approach is based on the array PQLs non-overlapped distribution that provides 

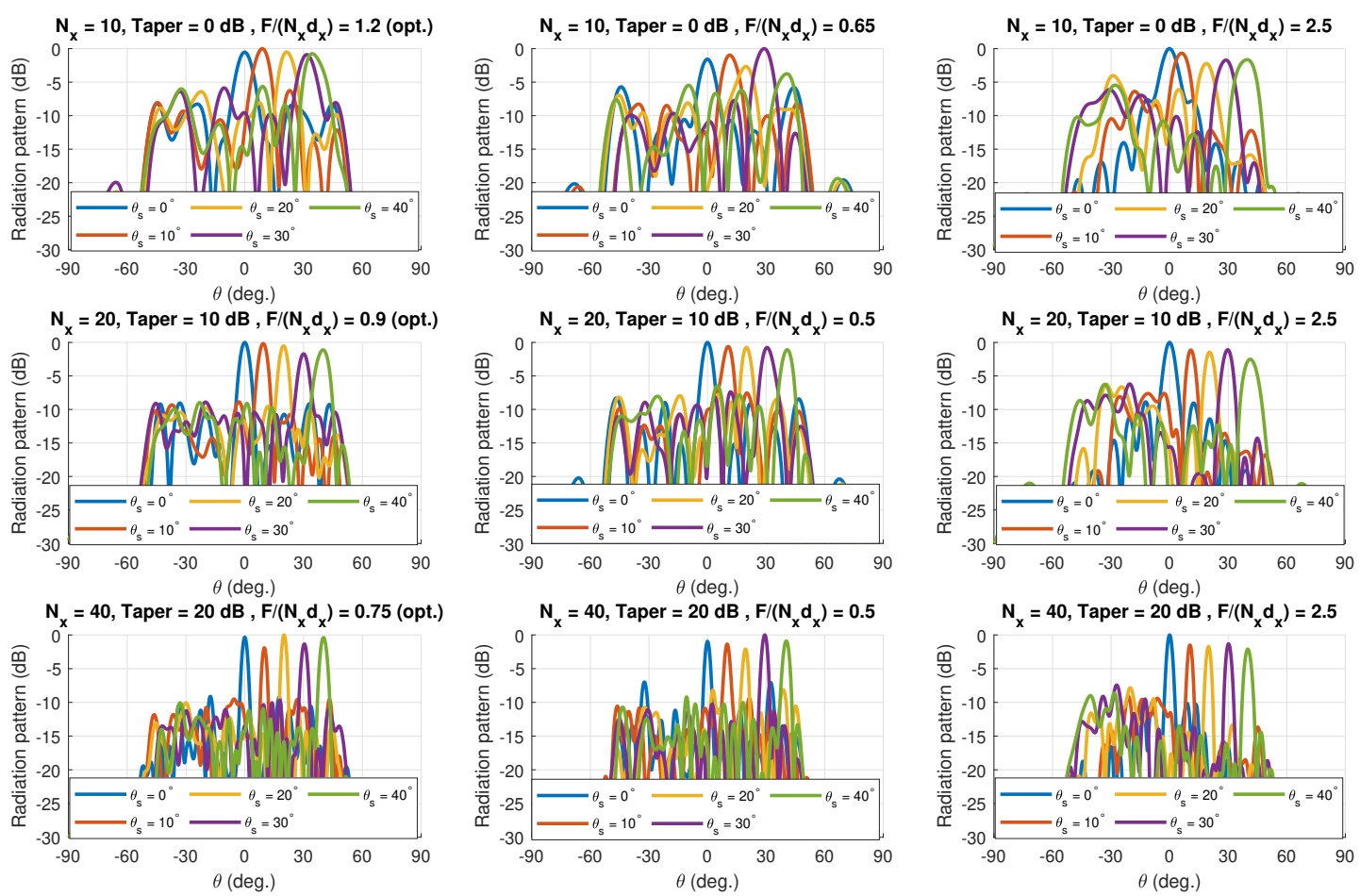

Fig. 6. Far-field patterns for different 1-bit phased array antennas within $0 \ldots 40^{\circ}$ beam-steering range (10 ${ }^{\circ}$ step). The left column corresponds to the optimum focal ratios as obtained from Fig. 3

"the best" phase quantization error randomization and realizes steerable radiation patterns with minimized maximum SLL. The main design relations for the optimum QO feed focal ratio were found analytically and verified numerically considering array channels amplitude distribution with an arbitrary taper. The proposed approach provides fairly accurate initial configuration of the QO feed. Further correction, of course, can be required to compensate for the edge and coupling effects as well as for non-perfect electrical performance of integrated PSs. The latter is a subject of future work. The extension of the method to the case of a 1-bit planar array (obtained by stacking of $N_{y}$ linear arrays) is quite straightforward if the amplitude and phase distributions can be presented as $A(x, y)=A_{x}(x) A_{y}(y), \varphi^{Q O}(x, y)=\varphi_{x}^{Q O}(x)+\varphi_{y}^{Q O}(y)$. The average power SLL is then $\propto 1 /\left(N_{x} N_{y}\right)$.

\section{ACKNOWLEDGMENT}

This work has received funding from the European Union's Horizon 2020 research and innovation programme under the Marie Skłodowska-Curie grant agreement No 860023 and the Sweden-Taiwan Collaborative Research Framework Project "Antenna Technologies for Beyond-5G Wireless Communication" from the Swedish Foundation for Strategic Research.

\section{REFERENCES}

[1] T. S. Rappaport, Y. Xing, O. Kanhere, S. Ju, A. Madanayake, S. Mandal, A. Alkhateeb, and G. C. Trichopoulos, "Wireless communications and applications above $100 \mathrm{GHz}$ : Opportunities and challenges for 6G and beyond," IEEE Access, vol. 7, pp. 78 729-78 757, Jul. 2019.
[2] M. C. Viganó, D. L. del Río, F. Bongard, and S. Vaccaro, "Sparse array antenna for Ku-band mobile terminals using 1 bit phase controls," IEEE Trans. Antennas Propag., vol. 62, no. 4, pp. 1723 - 1730, Apr. 2014.

[3] A. Clemente, L. Dussopt, R. Sauleau, P. Potier, and P. Pouliguen, "Wideband 400-element electronically reconfigurable transmitarray in X band," IEEE Trans. Antennas Propag., vol. 61, no. 10, pp. 5017-5027, Oct. 2013.

[4] A. R. Vilenskiy, M. N. Makurin, C. Lee, and M. V. Ivashina, "Reconfigurable transmitarray with near-field coupling to gap waveguide array antenna for efficient 2D beam steering," IEEE Trans. Antennas Propag., vol. 68, no. 12, pp. 7854-7865, Dec. 2020.

[5] A. Clemente, F. Diaby, L. D. Palma, L. Dussopt, and R. Sauleau, "Experimental validation of a 2-bit reconfigurable unit-cell for transmitarrays at Ka-band," IEEE Access, vol. 8, pp. 114991-114997, 2020.

[6] J. G. Yang, J. Lee, and K. Yang, "A W-band InGaAs PIN-MMIC digital phase-shifter using a switched transmission-line structure," in Proc. 2012 International Conference on Indium Phosphide and Related Materials, Santa Barbara, CA, USA, Aug. 2012, pp. 1-3.

[7] A. R. Vilenskiy, M. N. Makurin, and C. Lee, "Phase distribution optimization for 1-bit transmitarrays with near-field coupling feeding technique," in Proc. 14th European Conference on Antennas and Propagation, (EuCAP 2020), Copenhagen, Denmark, Mar. 2020, pp. 1-5.

[8] R. J. Mailloux, Phased array antenna handbook, 2nd ed. Norwood, MA: Artech House, 2018

[9] M. S. Smith and Y. C. Guo, "A comparison of methods for randomizing phase quantization errors in phased arrays," IEEE Trans. Antennas Propag., vol. 31, no. 6, pp. $821-828$, Nov. 1983.

[10] R. Maaskant, W. A. Shah, A. U. Zaman, M. Ivashina, and P.-S. Kildal, "Spatial power combining and splitting in gap waveguide technology," IEEE Microw. Wireless Compon. Lett., vol. 26, no. 7, pp. $472-474$, Jul. 2016.

[11] C. J. Miller, "Minimizing the effects of phase quantization errors in an electronically scanned array," in Proc. 1964 Symp. Electronically Scanned Phased Arrays and Applications, RADC-TDR-64-225, vol. 1, RADC Griffiss AFB, NY, USA, 1964, pp. 17-38.

[12] D. I. Voskresenskiy, L. I. Ponomarev, and V. S. Filippov, Convex scanning antennas: basic theory and analysis methods. Moscow: Soviet Radio, 1978, (in Russian). 